\title{
PERFIL OBSTÉTRICO E NEONATAL DOS PARTOS NATURAIS DOMICILIARES ASSISTIDOS POR ENFERMEIROS OBSTETRAS
}

\author{
OBSTETRIC AND NEONATAL PROFILE OF HOME CHILDBIRTH \\ DELIVERIES ASSISTED BY OBSTETRIC NURSES
}

\section{PERFIL OBSTÉTRICO Y NEONATAL DE LOS PARTOS NATURALES DOMICILIARIOS ASISTIDOS POR ENFERMEROS OBSTETRAS}

\author{
Reinaldo Dos Santos Moura* \\ Francisco JoIlsom Carvalho Saraiva** \\ Mylena Alves Santos*** \\ Ana Maria Rocha De Oliveira Santos**** \\ Regina Maria Dos Santos***** \\ Patricia Acioli De Barros Lima*****
}

\begin{abstract}
RESUMO
Objetivo: Descrever o perfil obstétrico e neonatal dos partos naturais assistidos por enfermeiros obstetras nos domicílios. Material y Método: Estudo documental de abordagem quantitativo-descritivo e retrospectivo, desenvolvido nos prontuários arquivados pelas duas únicas equipes de enfermagem obstétrica privada de Maceió, Alagoas. A coleta dos dados ocorreu entre abril a maio de 2017 e baseado na media estimada de 140; abordaram-se 72 prontuários disponíveis que cumpriram com todos os requisitos éticos de pesquisa deste cunho em relação à dispensa do termo de consentimento livre e esclarecido. Resultados: Com predomínio da faixa etária das genetrizes de 20 a 29 anos (58,3\%), casadas (58,7\%) e com nível superior (83,4\%). Multípara $(87,5 \%)$, com partos na água $(62,5 \%)$, de quatro apoios $(40,3 \%)$, sem laceração perineal $(77,8 \%)$ e com delivramento espontâneo (94,4\%). O aspecto do liquido amniótico claro (95,8\%), com Apgar e o teste do coraçãozinho satisfatório para manutenção da vida (100\%). Conclusão: Apesar das equipes de enfermagem obstétrica atuarem nos cenários hospitalar e domiciliar, o público procurou por partos domiciliares e evidenciouse que parir em casa é tão seguro do que parir institucionalmente e sobretudo se respeita a opinião feminina.
\end{abstract}

Palavras-chave: Parto Domiciliar; Parto Humanizado; Enfermagem Obstétrica; Características da População.

*Enfermeiro Gestor e Intensivista, Centro de Ensino Santa Juliana e Faculdade Seune. Maceió-AL, Brasil. Email: enfreinaldomoura@ gmail.com. Autor correspondente.

**Enfermeiro Obstetra, Centro Universitário Cesmac e Faculdade Seune. Maceió-AL, Brasil. Email: francisco.saraiva@cesmac.edu.br

***Enfermeira, Hospital Veredas de Alagoas. Maceió - AL, Brasil. Email: mylaa_alves@hotmail.com

****Enfermeira, Hospital Veredas de Alagoas. Maceió - AL, Brasil. Email: ana_santos@hotmail.com

*****Enfermeira Obstetra e Doutora, Universidade Federal de Alagoas (UFAL). Maceió - AL, Brasil. Email: relpesantos@gmail.com

******Enfermeira Obstetra, Mestra em Ensino na Saúde, Faculdade Seune e Universidade Estadual de Alagoas. Maceió-AL, Brasil.

Email: paciolifranca@gmail.com 


\section{ABSTRACT}

Objective: To describe the obstetric and neonatal profile of natural births assisted by obstetric nurses at home. Materials and Methods: Documentary study of quantitative-descriptive and retrospective approach, carried out using the medical records filed by the only two private obstetric nursing teams of Maceió, state of Alagoas, Brazil. Data collection took place between April and May 2017 and, based on an estimated average of 140, 72 available medical records that met all ethical research requirements in relation to the waiver of the informed consent form were used. Results: 72 records were analyzed, with a predominance of the age group of 20- to 29-year-olds (58.3\%), married (58.7\%) and having higher education level (83.4\%). Multicenter (87.5\%), water births $(62.5 \%)$, on four legs $(40.3 \%)$, no perineal laceration $(77.8 \%)$ and spontaneous placenta delivery (94.4\%). Clear amniotic fluid (95.8\%), APGAR and satisfactory heart test for life maintenance (100\%). Conclusion: Although obstetrical nursing teams work in the hospital and home settings, people preferred home births and it was shown that giving birth at home is as safe as giving birth institutionally, especially if it takes into account women's opinion.

Key words: Home Childbirth; Humanized Delivery; Obstetric Nursing; Population Characteristics.

\section{RESUMEN}

Objetivo: Describir el perfil obstétrico y neonatal de los partos naturales asistidos por enfermeros obstetras en los domicilios. Material y Método: Estudio documental de enfoque cuantitativo-descriptivo y retrospectivo, desarrollado con los registros archivados por los dos únicos equipos de enfermería obstétrica privada de la ciudad de Maceió, estado de Alagoas, Brasil. La recolección de datos fue entre abril y mayo de 2017 y se basó en una media estimada de 140. Finalmente se obtuvieron 72 fichas que cumplieron con todos los requisitos éticos de investigación respecto del consentimiento libre e informado. Resultados: Predominó el grupo de edad de las progenitoras de 20 a 29 ańos $(58,3 \%)$, casadas $(58,7 \%)$ y con nivel escolar superior (83,4\%). Multípara $(87,5 \%)$, con partos en el agua $(62,5 \%)$, de cuatro apoyos $(40,3 \%)$, sin laceración perineal $(77,8 \%)$ y con expulsión espontánea de la placenta $(94,4 \%)$. El aspecto del líquido amniótico claro $(95,8 \%)$, con APGAR y el test del corazón satisfactorio para el mantenimiento de la vida (100\%). Conclusión: A pesar de que los equipos de enfermería obstétrica actúan en los escenarios hospitalarios y domiciliarios, el público optó por partos domiciliarios y se evidenció que dar a luz en casa es tan seguro como dar a luz institucionalmente, sobre todo si se respeta la opinión femenina.

Palabras clave: Parto Domiciliario; Parto Humanizado; Enfermería Obstétrica; Características de la Población.

Fecha recepção: 05/03/2019

Fecha aceitação: 08/04/2019

\section{INTRODUÇÃO}

O estudo teve como objeto descrever o perfil obstétrico e neonatal dos partos naturais domiciliares (PND's) assistidos por equipes de enfermeiros obstetras (EO's) de Maceió Alagoas (AL). As justificativas acerca desta temática foram duas: a primeira, por compor a área de enfermagem em saúde da mulher e da criança e diante disso compreender que o enfermeiro é um dos profissionais habilitados da equipe de saúde, talvez o mais preparado, para lidar com o cuidado materno- infantil em todos os âmbitos da assistência, seja ela na atenção primária, secundária ou terciária; e a segunda, por ratificar a ausência de estudos nas principais bases de dados de enfermagem acerca deste objeto no recorte geográfico em apreço que caracterizasse a população estudada.

De acordo com o Conselho Federal de Enfermagem (COFEN) Brasileiro ${ }^{(1)}$ o enfermeiro obstetra possui atuação indispensável na assistência qualificada, desde pré-natal até o puerpério, por utilizar veementemente as práticas baseadas em evidências (PBE's), respeitando a autonomia dos 
atores sociais (mãe, recém-nascidos - RN's e demais familiares), com estratégias humanizadas e pautadas na qualidade com segurança do paciente, pois a mulher é preparada fisiologicamente para gerar e parir, enquanto os profissionais de saúde sáo meros coadjuvantes da assistência ${ }^{(2,3)}$.

Desde primórdios o parto foi considerado um evento fisiológico no ciclo de vida da mulher, vivenciado estritamente em caráter privadodomiciliar, que os saberes e as práticas das parteiras e de mulheres rezadeiras, eram suficientes para o momento parturitivo ${ }^{(4)}$. Contudo a partir do século $\mathrm{XX}$ a ciência biomédica transformou o parto natural (PN), de um evento fisiológico do binômio (mãe e filho) noutro evento, necessitando na maioria das vezes de tratamentos medicamentosos e cirúrgicos, levando a mulher para o ambiente institucionalizado, ou seja, transformando-os obrigatoriamente em institucionalizados ou partos hospitalares $(\mathrm{PH} \text { 's })^{(3,5)}$.

No Brasil estatisticamente o parto cesariano (PC) ainda é mais frequente serviços hospitalares (SH's), pois em alguns momentos, pela desinformação das mães, estes são induzidos de maneira arbitrária através de mitos de profissionais que visam apenas a rapidez no momento parturitivo e o lucros, porem mesmo assim algumas gestantes têm resgatado uma antiga prática natural de parir e hoje alguns profissionais obstetras valorizam o PN como um evento fisiológico ${ }^{(6,7)}$.

À partir da década 1980 foram criados no Brasil, pelo Ministério da Saúde (MS), o Programa de Assistência Integral à Saúde da Mulher (PAISM) e o Programa de Assistência Integral à Saúde da Criança (PAISC), ambos preconizando uma assistência humanizada e integral ao público alvo em todos os serviços do Sistema Único de Saúde $(\mathrm{SUS})^{(7,8)}$. Para se refletir sobre a atuaçáo moderna do enfermeiro obstetra (EO) na realidade brasileira, faz-se necessário compreender o processo de "construçáo" dessa profissão e o respeito a todos os programas ministeriais brasileiros ${ }^{(1,7,8)}$.

Ao resgatar a história do processo de parto, identifica-se em outrora a figura da parteira rural e urbana como responsável empiricamente pelo trabalho de parto, conhecendo o saber dessas mulheres, mas associando com as práticas baseadas em evidências (PBE's), caracterizam-se os EO's como profissionais capacitados para atuarem nos partos naturais (PN's) em qualquer cenário nas, desde que a gestação seja classificada como de baixo risco (GBR) de acordo com o Caderno de Atençáo Básica - 32 do MS de Atenção ao Pré-Natal de Baixo Risco e cobertura pela normatização de ${ }^{\circ}$ 0516/2016 do COFEN ${ }^{(1,5,6)}$.

$\mathrm{Na}$ capital alagoana existem até o momento duas equipes de EO's que atuam na assistência obstétrica em GBR apenas de forma privada, tanto no serviço hospitalar $(\mathrm{SH})$, quanto nos cenários extrahospitalares, respeitando, sobretudo a autonomia das mulheres e o bem estar do binômio ${ }^{(4,5,6)}$. Demonstra-se que as equipes de EO's reconhecem o seu papel diante do momento parturitivo, ao citar que são meros coadjuvantes, por adotar em sua prática o distanciamento da realidade biomédico do parto, na busca da antiga forma de parir, em cenários escolhidos por essas mulheres e familiares através no PN. Outro ponto importante a serem citadas que o público que busca os PND's é pessoas que já tinham vivenciado o parto institucionalizado, possuíam situações conjugal, escolar e financeira estáveis, ou seja, sabiam o que queria ${ }^{(1,4,5,6)}$.

Reconhece a importância e a relevância deste estudo ao destacar que as informaçóes sobre as particularidades dos nascimentos, em qualquer cenário, são relevantes para o Brasil e o recorte geográfico em apreço, pois tanto as informaçóes maternas-perfil obstétrico, quanto as informaçôes do bem estar dos RN's-perfil neonatal, numa determinada localidade e período, são relevantes, por permitir a elaboração de indicadores demográficos e epidemiológicos que podem representar a realidade da comunidade para o planejamento de estratégias de melhorias das condiçōes de saúde, com o intuito do exercício das $\mathrm{PBE} \mathbf{s}^{(4,5,6)}$ e da exteriorização de que é seguro e humano parir em casa para o binômio ${ }^{(6)}$.

Á partir o que foi externado acima, baseado na importância, relevância e nas justificativas do estudo, adota-se como ponto de partida a inquietação que se impôs de certa forma a conduzir o estudo: qual o perfil obstétrico e perinatal dos PND's assistidos por equipes de EO's? Baseado em literaturas referidas ${ }^{(1,4-6)}$, elaborou-se como hipótese "que a maioria das mulheres que optaram pelos PN's com os EO's possuía situação conjugal estável, um elevado nível escolar e preferiram os cenários domésticos para o momento perturitivo". Diante disso, objetivou-se: Descrever o perfil obstétrico e neonatal dos PND's assistidos por equipes de EO's. 


\section{MATERIAL E MÉTODO}

Estudo documental de abordagem quantitativodescritiva e retrospectiva ${ }^{(9)}$, desenvolvido nos prontuários arquivados pelas as duas únicas equipes privadas de EO’s de Maceió - AL, para fins de conclusão do Curso de Graduação em Enfermagem.

As equipes inseridas na pesquisa são compostas por EO's, com ambulância de suporte avançado e um profissional médico de sobreaviso, para qualquer intercorrências. Eram os únicos no recorte temporal e geográfico do estudo, atendem em todo o estado e prestam assistência de enfermagem nos PN's nos diferentes cenários escolhidos pela parturiente. Como forma de resguardar os princípios de confidencialidade, esses grupos foram nomeados por nomes de deusas associadas à maternidade egípcia e grega, tais quais: Ísis e Ilítia, o grupo Ísis, atende há três anos apenas nos partos naturais domiciliares (PND's), já o grupo Ilítia atende há dois, tanto nos PND's, quanto nos $\mathrm{PN}$ em SH.

A amostra foi calcula maximizando a variância de $50 \%$, visto que não existia informação prévia a respeito desta estimativa em Alagoas, portanto foi considerado um nível de confiança de 95\% e um erro máximo de 5\% com base na Fórmula: $\mathrm{N}=(1,645) 2 \mathrm{P}(1-\mathrm{p}) / 0,12^{(9)}$, baseado na media estimada de 140 prontuários, calculou-se a amostra de 94. Porém alguns critérios foram eleitos para o melhor andamento da pesquisa, o primeiro de inclusão: prontuários completos e os de exclusão: prontuários rasurados, ilegíveis ou não disponíveis na fase de coleta dos dados, determinando assim um total de 72 prontuários disponíveis.

A coleta dos dados ocorreu entre os meses de abril á maio de 2017, baseada nas variáveis eleitas para o estudo e no instrumento utilizado noutra pesquisa $^{(4)}$. No primeiro momento foi escolhido para caracterizar os dados sociodemográficos das parturientes e dos RN's quando couber: o sexo, escolaridade, faixa etária, etnia, estado civil, região de procedência e profissão.

As variáveis para o perfil obstétrico foram divididas em três categorias, tais quais: a primeira, antecedentes obstétricos (número de gestação, de paridade e as via de parto); a segunda, relacionada ao pré-natal (início do pré-natal, número de consultas do pré-natal com o EO e o tipo de acompanhante nas consultas); e a terceira, ressaltando o momento parturitivo (acompanhante, posição do parto, apresentação fetal, integridade perineal, tempo de rompimento das membranas ovulares, tipo de rotura das membranas ovulares, aspecto do líquido amniótico, delivramento e encaminhamento ao hospital). Já para a coleta do perfil neonatal do RN foram considerados os escores de Apgar ${ }^{(5)}$ e do teste do coraçãozinho ${ }^{(10)}$.

Construiu-se um banco de dados baseados nessas variáveis no Microsoft Excel $2010^{R}$ e foi realizada à análise estatística descritiva-percentil, com o software Statistical Package for Social Science (SPSS) versão 20.0 e Statistical Analisys System 9.02, explicitados os resultados entre as frequências absolutas e relativas através de tabelas ${ }^{(9)}$.

Respeitaram-se os princípios bioéticos da Resolução 466/2012 do Conselho Nacional de Saúde no que diz respeito às pesquisas deste cunho quanto à dispensa do termo de consentimento livre e esclarecido, esta foi aprovada pelo Comitê de Ética e Pesquisa da Universidade Federal de Alagoas através da Plataforma Brasil, no dia 26 de janeiro de 2017, sob o parecer de no 1.899 .671 e o CAAE: 59662316.0.0000.5013.

\section{RESULTADOS}

Abordaram-se 72 prontuários e verificou que o perfil de atendimento das equipes de EO's, em relação à procura do público, possuía divergência quanto ao tempo de atuação e a quantidade dos atendimentos realizados, pois o Grupo Ilítia o mais recente, possui uma quantidade maior de assistência no PN (40 / 55,6\%), quando comparado ao pioneiro Ísis (32 / 44,4\%), sobretudo o Grupo Ísis atua exclusivamente com os PND's, mesmo todos os ter sidos assistidos no cenário domiciliar por ambos.

$\mathrm{Na}$ caracterização das genetrizes quanto ao perfil social e demográfico (Tabela 1) observou-se que na houve um predomínio de que a maioria das genetrizes possuíam estado civil entre casas e uniôes estáveis (52), com faixa etária considerada produtiva de 20 à 29 anos (42), com ensino superior (60), funcionárias públicas (59) e da região nordeste (65). Na distribuição dos antecedentes obstétricos (Tabela 2), nota-se que a maioria das genetrizes foram classificadas como multigesta (45) e multípara (63), com partos por via cesariano (58) e em hospitais (72). Abordando a primeira distribuição do perfil obstétrico em relação às 
consultas de pré-natal (Tabela 3), ressalta-se a o número acima $>9$ consultas (68) e o do esposo preponderância das taxas do início da consulta de como acompanhante neste momento (69). pré-natal com o enfermeiro em 12 semanas (68),

Tabela 1. Caracterização do perfil social e demográfico das genetrizes (n=72). Maceió (AL), Brasil, 2017.

\begin{tabular}{llrr}
\hline Variável & & N & \% \\
\hline Estado cível & União Estável & 10 & 13,7 \\
& Solteira & 20 & 27,8 \\
Faixa etária & Casada & 42 & 58,7 \\
& $19-19$ anos & 1 & 1,4 \\
& $20-29$ anos & 42 & 58,3 \\
Escolaridade & $30-45$ anos & 29 & 40,3 \\
\multirow{3}{*}{ Profissáo } & Ensino Médio & 12 & 16,7 \\
& Ensino superior & 60 & 83,4 \\
& Contadora & 1 & 1,4 \\
& Assistente Social & 3 & 4,2 \\
& Professora & 4 & 5,6 \\
Naturalidade por regiōes & Enfermeira & 5 & 6,9 \\
& Funcionária Pública & 59 & 81,9 \\
& Sudeste & 1 & 1,4 \\
& Nordeste & 6 & 8,3 \\
\hline
\end{tabular}

Tabela 2. Distribuição dos antecedentes obstétricos das genetrizes (n=72). Maceió, Brasil, 2017.

\begin{tabular}{llrr}
\hline Variável & & N & \% \\
\hline Gestaçáo & Uma & 9 & 12,5 \\
& Duas & 18 & 25 \\
\multirow{3}{*}{ Paridade } & Três & 45 & 62,5 \\
& Segundípara & 9 & 12,5 \\
Via do parto hospitalar & 63 & 87,5 \\
& Multípara & 5 & 6,9 \\
& Ambos (Natural e Cesariana) & 9 & 12,5 \\
& Natural/Vaginal & 58 & 80,6 \\
\hline
\end{tabular}

Tabela 3. Distribuição do perfil obstétrico relacionado ao pré-natal com o grupo de enfermeiro ( $\mathrm{n}=72)$. Maceió, Brasil, 2017.

\begin{tabular}{llrr}
\hline Variável & & N & $\%$ \\
\hline Início do pré-natal & 12 semanas & 68 & 94,4 \\
& 12 a 20 semanas & 4 & 5,6 \\
\multirow{2}{*}{ Número de consulta } & 6 a 9 & 4 & 5,6 \\
\multirow{2}{*}{ Acompanhante } & $>9$ & 68 & 94,4 \\
& Mãe & 3 & 4,2 \\
& Esposo & 69 & 95,8 \\
\hline
\end{tabular}


Examina-se na Tabela 4 a prevalência durante o momento o parturitivo do perfil obstétrico, do acompanhante esposo (60), com o parto na água (45), na posição quatro apoios (29), da apresentação fetal cefálica (57), sem laceração do períneo (56), do tempo curto e de maneira espontânea (57), de rompimento das membranas ovulares (70), do aspecto claro do líquido amniótico (69), do delivramento espontâneo (68) e do não encaminhamento do binômio ao hospital, pela inexistência de intercorrência (69). O perfil neonatal é trazido na Tabela 5 com a adaptação do RN ao meio com saúde e qualidade de vida. Observa-se um predomínio dos RN's em contato com a pele da mãe no primeiro minuto (58), da maioria enquadrado no escore de Apgar no primeiro minuto de 7 a 8 (58), no quinto minuto entre 8 a 9 (72) e no décimo minuto com 9 (68). Já em relação ao escore do coraçãozinho nas primeiras 24 horas de vida, os resultados do membro superior direito (MSD) todos estavam (72), entre o escore de 95 a $97 \%$ de saturação e nos inferiores entre 94 a $95 \%$ de saturação, ambos em oximetria digital portátil. Outro ponto foi que a maioria teve sucção efetiva (68).

Tabela 4. Distribuição do perfil obstétrico durante o momento parturitivo (n=72). Maceió, Brasil, 2017.

\begin{tabular}{|c|c|c|c|}
\hline Variável & & $\mathbf{N}$ & $\%$ \\
\hline \multirow[t]{3}{*}{ Acompanhante } & Filhos & 1 & 1,4 \\
\hline & Mãe & 11 & 15,3 \\
\hline & Esposo & 60 & 83,3 \\
\hline \multirow[t]{2}{*}{ Local do parto } & Fora da água & 27 & 37,5 \\
\hline & Água & 45 & 62,5 \\
\hline \multirow[t]{5}{*}{ Posição do parto } & Em pé & 1 & 1,4 \\
\hline & Lateral & 4 & 5,6 \\
\hline & Sentada na Banqueta & 16 & 22,2 \\
\hline & Cócoras & 22 & 30,6 \\
\hline & Quatro Apoios & 29 & 40,3 \\
\hline \multirow[t]{2}{*}{ Apresentação fetal } & Pélvico & 15 & 20,8 \\
\hline & Cefálico & 57 & 79,2 \\
\hline \multirow[t]{2}{*}{ Integridade perineal } & $\begin{array}{l}\text { Laceração } 1^{\circ} \text { grau sem } \\
\text { episiorrafia }\end{array}$ & 16 & 22,2 \\
\hline & Sem laceração & 56 & 77,8 \\
\hline \multirow[t]{3}{*}{ Tempo de rompimento das membranas ovulares } & $>3$ a 6 horas & 70 & 97,2 \\
\hline & 6 a 17 horas & 1 & 1,4 \\
\hline & $>18$ horas & 1 & 1,4 \\
\hline \multirow[t]{2}{*}{ Tipo de rotura das membranas ovulares } & Artificial & 15 & 20,8 \\
\hline & Espontânea & 57 & 79,2 \\
\hline \multirow[t]{2}{*}{ Aspecto do líquido amniótico } & Mecônio & 3 & 4,2 \\
\hline & Claro & 69 & 95,8 \\
\hline \multirow[t]{2}{*}{ Delivramento } & Manual & 4 & 5,6 \\
\hline & Espontâneo & 68 & 94,4 \\
\hline \multirow[t]{2}{*}{ Encaminhamento ao hospital no caso de intercorrência } & Sim & 3 & 4,2 \\
\hline & Não & 69 & 95,8 \\
\hline
\end{tabular}


Tabela 5. Distribuição do perfil neonatal (n=72). Maceió, Brasil, 2017.

\begin{tabular}{llrrr}
\hline Variável & & & N & $\%$ \\
\hline Contato com a pele da mãe no primeiro minuto & Não & & 14 & 19,4 \\
& Sim & & 58 & 80,6 \\
Escore de APGAR & Primeiro minuto & 6 a 7 & 14 & 19,4 \\
& & 7 a 8 & 58 & 80,6 \\
& Quinto minuto & 8 a 9 & 72 & 100 \\
& Décimo minuto & 9 & 68 & 94,4 \\
Escore do teste do coraçãozinho & & 10 & 4 & 5,6 \\
& Membro superior & 93 a $95 \%$ & 72 & 100 \\
Amamentação na primeira hora & direito & 94 a 95\% & 58 & 80,6 \\
& Membros inferiores & 96 a $98 \%$ & 14 & 19,4 \\
& & & 4 & 5,6 \\
\hline
\end{tabular}

\section{DISCUSSÁO}

De acordo com um estudo realizado também na região nordeste do Brasil ${ }^{(11)}$, apontou que a opção pelos partos hospitalares (PH's), seja ele $\mathrm{PN}$ ou PC, normalmente é pela cultura de se achar que o ambiente institucional é mais seguro, talvez explique a procura destas primeiro pelo parto institucional biomédico, como visto neste estudo no que concerte aos partos institucionais. Também, um estudo inglês ${ }^{(12)}$ apontou que as maiorias das mulheres submetidas, primeiro aos $\mathrm{PH}$ 's, ratificaram que são levadas para o ambiente institucional pelo do próprio conhecimento do senso comum e a imposição do sistema biomédico, como se o parto fosse algo arriscado para a saúde do binômio.

Contudo, noutro estudo realizado em Florianópolis $^{(4)}$ e outro brasileiro ${ }^{(7)}$ convergiu com este estudo, por apontar a preferencia das parturientes por este cenário parturitivo, ainda aqui tal estudo ratifica que a atuação dos EO's nos PND's estimula o benefício do empoderamento feminino e para o casal por considerar que este parto é uma forma de recepcionar de maneira mais fisiológicanatural e com qualidade o binômio ${ }^{(4,5)}$.

Quanto ao perfil social e demográfico deste estudo, alguns dos dados convergiram com outro estudo $^{(4)}$, em relação que a maioria das mulheres que pariram através dos PND's estarem em relacionamentos estáveis, na faixa etária produtiva, com um grau de escolaridade elevado (nível superior), caracterizada profissionalmente como funcionária pública e com o público de atendimento da mesma regiáo natural do grupo de $\mathrm{EO}$, mesmo este estudo tendo sido desenvolvido noutro recorte geográfico brasileiro, ratificando assim a hipótese do estudo.

Em outros estudos com percurso metodológico diferente deste ${ }^{(2,3,7)}$, a opção de mulheres pelos PND's com fator de segurança e conforto, referiu que tal escolha foi devido ao nível elevado escolar destas mulheres, pela seu grau de conscientização da fisiologia do parto e pelo seu próprio empoderamento feminino. Conforme indicado em um estudo de Goiânia $^{(12)}$ e outros ${ }^{(3,4,6)}$ a maioria das mulheres que optam pelo PN com os EO's foram pessoas que tiveram experiências negativas anteriores, pelo reconhecimento do hospital não ser um cenário seguro e humanizado, e seus domicílios denotam confiança e a equipe que a acompanhasse respeitaria sua autonomia durante todo o processo parturitivo.

A melhor maneira de entender o parto humanizado é destacar as dificuldades de sua implantação em maternidades e hospitais ${ }^{(8)}$, uma vez que a autonomia do casal no ambiente hospitalar 
do SUS está longe de ser executada e respeitada, por inúmeras desculpas como déficits financeiros, recursos estruturais e humanos no setor da saúde e também por profissionais que confundem humanização com pequenos cuidados ${ }^{(8)}$.

Em relação ao antecedente de grandes números de PC, outros autores ${ }^{(11,13)}$ corroboram com que foi apontado pelo o Caderno de Atenção Básica - 32 do Ministério da Saúde de Atenção ao Pré-Natal de Baixo Risco ${ }^{(5)}$, ao referir que paradoxalmente a corrente por partos cesarianos, desde o ano de 1999 o MS vem incentivando numa campanha a desconstrução de que o PC deve ser a primeira escolha nos hospitais pela equipe médica, enfatizando que este só deve ser utilizado se houvesse algum risco de vida para o binômio.

Novamente trazendo à baila novamente as informaçóes do Caderno $32^{(5)}$ ratifica que o objetivo do pré-natal é "assegurar o desenvolvimento da gestação, permitindo o parto de um recém-nascido saudável, sem impacto para a saúde materna, inclusive abordando aspectos psicossociais e as atividades educativas e preventivas". Ainda aqui explicita informações que corroboram com este estudo no primeiro perfil obstétrico abordado, relacionado à atenção do pré-natal, ao abordar que este deve ser iniciado mais precocemente na $12^{\mathrm{a}}$ semana, com no mínimo 6 consultas, que pelo menos duas com o profissional médico e que todo o período gestacional e partutitivo a mulher tem direito a um acompanhante resguardado pela lei brasileira de $\mathrm{n}^{\mathrm{o}} 11.108$, de 7 de abril de $2005^{(5)}$.

Em relação a variável acompanhante, noutro estudo realizado na Holanda ${ }^{(14)}$ apontou que o acompanhante no momento parturitivo encoraja e apoia as parturientes, em até certo ponto diminui a dor do parto e consideravelmente a mulher se sente mais acolhida. Assim o EO deve incluir a figura paterna em todo o planejamento do prénatal, nos cuidados do trabalho de parto, no parto e nascimento do recém-nascido (RN) ${ }^{(5,7,14)}$, pois os homens assim como as mulheres são afetados emocionalmente pela gravidez, na tentativa de compreender suas expectativas, responsabilidades, medos e emoções. Tais informações também entram consonância com outro estudo realizado em Santa Catarina ${ }^{(15)}$, com amostra documental de 212 prontuários abalizou que a prevalência do inicio precoce do pré-natal (12a semana), o mínimo de 6 consultas com o enfermeiro obstetra e duas com o profissional médico obstetra e que as consultas na maioria acompanhadas por seus conjugues, mesmo o recorte temporal e a amostra sendo distinta deste, ainda assim se observa harmonia com nossos resultados. Quanto mais cedo se iniciar as consultas de pré-natal (mínimo 8) e a manutenção da periodicidade destas durante o período gestacional até o parto, diminuiriam ou zeraria riscos previsíveis ao binômio ${ }^{(16,17)}$.

Observa-se corroboração com outras evidencias $^{(15)}$ até certo ponto com o perfil obstétrico no momento parturitivo, em relação da maioria dos PND's terem sido na água, com apresentação do líquido amniótico de cor clara e com um número reduzido de intercorrências com o binômio que necessitasse transferência hospitalar. Mais todavia alguns dados foram divergentes, tais quais: o alto índice de laceração perineal, o tempo acima de 18 horas para o rompimento das membranas ovulares e com isso levou a ruptura destas membranas de maneira artificial e a taxa elevada dos delivramentos manuais, sobretudo os demais resultados não foram abordados no estudo destes autores ${ }^{(14)}$.

Abordando ainda a questão da integridade perineal durante o momento parturitivo, uma revisão integrativa produzida por EO's portuguesas e que descreveu as estratégias que podem ser desenvolvidas pela parteira na preservação do trauma perineal, durante o segundo período do trabalho de parto $^{(2)}$, aponta uma explicação pertinente baseada nas PBE's, que para o número mínimo de laceração deste estudo (Tabela 4), o enfermeiro obstetra, desde consultas de pré-natal, preparou a gestante com exercício físico no geral, massagens e exercícios locais e tratamentos não farmacológicos perineais, reduzindo assim o risco de traumas lacerantes, explicando com isso, talvez, o elevado número de consultas de pré-natal da Tabela 3 propostas pelas literaturas $^{(1,5)}$. Corroborando com revisiones ${ }^{(2)}$ e com no que concerte a laceração perineal e ao delivramento espontâneo de este estudo (Tabela 4), um ensaio clínico randomizado norte americano ${ }^{(18)}$, explicitou que as mulheres que foram submetidas ao tratamento de crioterapia em todas as fases do parto pariram mais rápido e com isso preservou-se a integridade perineal e quando teve laceração foram apenas as mas leves, diferentes daquelas receberam tratamento farmacológico por analgesia.

Em consonância com o que foi visto neste estudo (Tabela 4) e com a literatura ${ }^{(5,8,15,16,17,18)}$, a $\mathrm{OMS}^{(19)}$ 
indica 56 recomendaçóes sobre os cuidados com o binômio, da gestação ao puerpério, ao enfatizar o direito do binômio as questôes básicas, do direito ao acompanhante durante o trabalho de parto, e da opção de tomada decisória da mulher no gerenciamento de sua dor e, no cenário parturitivo, de sua escolha numa posição eleita também por ela e não pelo profissional assistente, sobretudo ao respeito pelo seu desejo de um parto totalmente natural até a fase de expulsão.

De acordo com outros estudos ${ }^{(5,15,20)}$ o número elevado de consultas preconizadas pelo MS no pré-natal e o início precoce deste, eles som para questôes como: a preferencia das parturientes pela água como local de parição, a posição de quatro apoios no momento expulsivo, a ausência de qualquer distócia e a prevalência da apresentação fetal cefálica nos PND's.

Num estudo realizado em Tanzânia ${ }^{(21)}$ apontou dados que convergiram com os resultados deste, ao abalizar que os profissionais de saúde são qualificados para prestarem a assistência ao binômio. Todavia o sistema biomédico impóem regras que afastam essas mulheres do cenário institucional, como por exemplo, a própria posição de parir não fisiológicaginecológica e litotômica.

A opção de mulheres pelos PND's com fator de segurança e conforto ${ }^{(22)}$, aponta que a posição litotômica é mais fácil para o profissional médico mais não para a fisiologia dos $\mathrm{PN}$ e que foi introduzida neste ambiente pelo modelo hegemônico de saúde. Nos PND's existe para a parturiente a liberdade de escolha pela melhor posição e local para parir, que aqui se menciona como local a água e posição cócoras e quatro apoios, demonstrando que se pode parir na posição escolhida por essas. Assim redesenhou-se uma nova perspectiva de parto $^{(7)}$ para os cuidados com o binômio ${ }^{(5,19)}$.

$\mathrm{O}$ ensino de cuidados com a gestante pelo acompanhante vão desde o período gestacional até o pós-puerpério, e este deve participar de todo o processo primeiramente, utilizando além da sua presença as terapias não farmacológicas (massagens, exercícios respiratórios, a prática do diálogo e o estímulo à deambulação), ou seja, preparando este como um assistente durante o momento gestacional $^{(23)}$. Os EO's defendem ao seu favor o desanuviamento do casal, para que esses possam opinar e escolher o que melhor convém naquele momento para a promoção do próprio bem-estar, livres de quaisquer amarras biomédica e coercitiva, pois dentro desta perspectiva dizemos que o acesso às informações faz das mulheres empoderadas.

Os EO's desempenha uma função crucial na assistência obstétrica humanizada, porque na sua formação desde graduação até a especialização obstétrica, foi preparado para o cuidar pautado no atendimento integral do binômio e da família, respeitando a fisiologia do parto natural em qualquer cenário escolhido pelos atores sociais, já que neste ambiente os profissionais de saúde são meros coadjuvantes $(2,5,11,19)$.

Para se tiver um avanço na humanização da assistência no processo de nascimento $\left({ }^{21)}\right.$, é necessário investir em açóes que modifiquem a mentalidade do pessoal em relação ao cuidado maternal-infantil digno, que a própria unidade hospitalar se adapte as normas da $\mathrm{OMS}^{(19)}$ como um todo e que se invista em treinamento continuado de pessoal em relação a sua cultura de trabalho.

O PND é considerado um momento de alto clímax emocional para os envolvidos (mãe, pai, família e RN), deste modo o ambiente parturitivo de possuir características harmoniosas com as $\mathrm{PBE}^{\prime} \mathrm{s}^{(7)}$. Dentro deste ponto de vista a equipe obstétrica é coadjuvante que devem se atentar para todos os momentos e contextos daquele ambiente, todavia ser sensível e atencioso com todos, sobretudo o cuidado maior de acordo com os país são com os $\mathrm{RN}^{\prime}{ }^{(7)}$.

Levando em consideração tal abordagem para o PND ser considerado um sucesso, além dos cuidados observados com o perfil obstétrico, e necessário que os RN's esteja com saúde e protegido no colo familiar, como visto neste estudo (Tabela 5), ao apontar o perfil satisfatório obstétrico em relação ao nascimento com saúde e segurança para o $\mathrm{RN}^{(5,8,19)}$.

Observando os resultados do escore de Apgar noutro estudo ${ }^{(20)}$, pode-se explicitar harmonia com este estudo (Tabela 5), ao assinalar que o escore evoluiu positivamente desde o primeiro minuto até o quinto, não obtendo a nota máxima em nenhuma avaliação. Todavia como fator limitante neste estudo foi a ausência da última avaliação dos primeiros 10 minutos de vida e a não execuçáo do teste do coraçãozinho.

Ressalva-se que no enquadramento do Apgar, de acordo com as literaturas referidas oficial ${ }^{(5,19)}$, aborda-se que os RN's estavam na sua grande 
maioria sem asfixia ou com qualquer tipo de parada cardiorrespiratória, ou seja, tudo transcorria de maneira positiva e evolutiva, quando se aborda o perfil neonatal.

A partir de 2014 surgiu uma portaria de $n^{\circ}$ 20, de 10 de junho, tornado público a decisão de incorporar a oximetria de pulso-teste do coraçãozinho, que considera o resultado normal a saturação periférica maior ou igual a $95 \% \mathrm{em}$ ambas as medidas (MSD e MMII), com a menor que 3\% entre as medidas do MSD e os $\mathrm{MMII}^{(10)}$. De acordo com neonatologistas norte americanos e que descreveu a prática atual e esclareceu a opiniáo destes com relação ao rastreamento das patologias congênitas com relação a oximetria de pulsoteste do coraçãozinho ${ }^{(24)}$ e aqui referiu da eficácia positiva de quase oitenta porcento para o rastreio, classificando-a como uma ferramenta simples e segura.

Abordando o contexto de segurança do paciente (binômio) e do comprometimento da equipe de EO envolvida nos PD's em sempre buscar melhorias pautadas nas PBE's, outro estudo brasileiro( ${ }^{(25)}$, realizado em Campinas - SP, que identificou quais eram as boas práticas que fazem parte da assistência ao parto em domicílio na voz de mulheres que tiveram o parto em casa, corroboraram com este estudo e com outro estudo internacional ${ }^{(24,26)}$, justamente pela preferencia das equipes de saúde optarem pela cientificidade e humanização no parto.

De acordo com outro construto inglês que comparou e explorou os resultados perinatais e maternos com mulheres com "maior risco" de complicaçóes que planejaram os PNDs versus $\mathrm{PH}^{(26)}$, apontou que as mulheres classificadas com GBR quando pariram nos seus domicílios ou em qualquer ambiente extra-hospitalar, os RN's não eram afastados de suas genitoras no período perinatal, ou seja, nenhuma intercorrências ocorria com o binômio nos PND's; tal dado corrobora com este estudo (Tabela 4 e 5) e com outras produçôes já mencionados ${ }^{(6,15,20)}$.

Trazendo ainda à tona a triagem do coraçãozinho, outro estudo realizado numa realidade distinta desta $^{(27)}$, ratificou que 95 por cento dos RN's submetidos a tal triagem, não possuíam patologias congênitas cardíacas e nem foram necessários realizar as manobras de reanimação cardiopulmonar, corroborando assim com este estudo (Tabela 5), pois nenhum deste aqui nasceu com patologia congênita cardíaca e nem foram expostos ao sofrimento durante o nascimento de acordo com o escore de literaturas oficiais ${ }^{(5,10,19)}$.

O contato pele corpo do binômio é importantíssimo para o estreitamento do vinculo e humanização no parto; tal resultado foi visto neste estudo (Tabela 5) e corroborou também com outros ${ }^{(5,15)}$, que apontam que os PND's são considerados humanizados e seguros por contemplar a ascensão do binômio.

Para alguns autores ${ }^{(7,8)}$ o respeito e a sensibilização do cuidado são mecanismos cruciais para satisfação dos atores dos PND's, devendo todos os profissionais envolvidos com cuidado materno-infantil assumirem o papel de mero coadjuvante no cotidiano de trabalho. Assim se ratifica que a família ao buscar um estilo de parto contra hegemônico no Brasil, contrapóe-se ao modelo frio e tecnocrático, valorizando exacerbadamente vivencia do parir e nascer numa metamorfose sentimental ${ }^{(23)}$.

$\mathrm{Na}$ mesma pesquisa realizada em Campinas $\mathrm{BR}^{(25)}$, corroborou com este estudo (Tabela 5) e com outros ${ }^{(7,15)}$, ao citar que o casal ao recepcionar o RN quer disponibilizar toda forma de qualidade e segurança para este, a tal ponto que um dos primeiros posicionamentos é contato pele com pele do binômio, favorecendo assim o vinculo precoce e que a amamentação precoce tem a finalidade do mais sublime respeito cultural e de saúde do cuidado materno-infantil. Enfatiza-se que tal variável acerca da amamentação durante as primeiras horas foi encontrada apenas numa única literatura ${ }^{(20)}$.

A qualidade da assistência prestada ao binômio reflete pontualmente na temática de segurança do paciente, pois esta é sugerida baseada nos resultados de destaque das informaçóes sobre as particularidades dos nascimentos, assim as mudanças que ocorrem além do desenvolvimento de infraestrutura, da disposição e defesa de mudanças com treinamento das equipes de saúde, com a finalidade de zerar a mortalidade materna, neonatal e infantil, é o ponto crucial para o incentivo de estudos como este ${ }^{(28)}$.

De acordo com o conceito "parto humanizado", os EO's são formados para o cuidado maternoinfantil dentro de uma perspectiva de assistência que valoriza além da saúde e o bem estar do binômio, outros aspectos holísticos (físicos, psíquicos, sociais, culturais e espirituais ${ }^{(29)}$, são profissionais habilitados para atuarem nos partos em qualquer 
cenário desde que a GBR. Assim, de uma maneira ampla e geral, a análise conceitual acerca dos PN's de forma humanizada explicita o respeito que a equipe de saúde possui ao protagonismo e autonomia das mulheres desde via de parto, ao acompanhamento e o respeito as $\mathrm{PBE}{ }^{\prime(29)}$.

Em relação ao bem estar do binômio do período gestacional até o perinatal, de acordo com um estudo holandês ${ }^{(30)}$, apontou que não foi encontrado aumento dos riscos adversos no período em apreço para os PND's; contudo se enfatizou que estes resultados foram aplicados apenas em regióes onde os PND's são bem integrados ao sistema de assistência de saúde, corroborando assim com os dados gerais deste estudo no que concerte que parir em casa é táo seguro do que no ambiente institucional para o binômio.

Destaca-se que são necessários mais esclarecimentos acerca deste objeto, pois se observou alguns fatores limitantes neste estudo: o fator o tempo do estudo por ser um trabalho de conclusão do curso de graduação em enfermagem; o próprio delineamento metodológico e a falta de informaçóes científicas com a mesma temática no recorte geográfico em apreço.

\section{REFERÊNCIAS}

1. Brasil. Conselho Federal de Enfermagem-COFEN. Resolução 0516/2016. Disponível em: http://www. cofen.gov.br/resolucao-cofen-no-05162016_41989. html

2. Ferreira-Couto CM, Fernandes-Carneiro MN Prevenção do traumatismo perineal: uma revisão integrativa da literatura. Enferm Global. 2017 jul; 47: 552-563. Disponível em: https://revistas. um.es/eglobal/article/view/252131

3. Oliveira JC, Paula AC, García ESGF, Andrade MBT, Leite EPRC. Assistência obstétrica no processo de parto e nascimento. Rev Fund Care Online. 2018 abr; 10(2): 450-457. Disponível em: http://www. seer.unirio.br/index.php/cuidadofundamental/ article/view/6083/pdf_1

4. Feyer ISS, Monticelli M, Knobel R. Perfil de casais que optam pelo parto domiciliar assistido por enfermeiras obstétricas. Esc Anna Nery. 2013 abr-jun; 17(2): 298-305. Disponível em: http://

\section{CONCLUSÁO}

Em relação ao perfil obstétrico se observou que durante o período do pré-natal as mulheres assistidas por EO's fizeram mais consultas do que o preconizado pelo MS e com o inicio precoce deste, acompanhadas por seus esposos durante e depois do parto. $\mathrm{O}$ momento parturitivo foi considerado rápido e sem intercorrências para o binômio, com a preferencia das mulheres por partos na água, sem episiorrafia e na posição de quatro apoios, com delivramento placentário natural, rompimento da membrana ovular espontânea e aspecto claro do liquido amniótico.

Os PND's também foram considerados seguros e com ambientes emocionalmente propícios para criação do vinculo para o binômio, já que se teve o contato pele a pele no primeiro minuto e a amamentação nas primeiras horas de vida. Os resultados positivos da escala de Apgar e do teste do coraçãozinho reforçou que o cenário domiciliar é tão seguro quanto o hospitalar para os RN's e mães classificadas com GBR. Espera-se que mais estudos sejam realizados a partir deste e que o paradigma de segurança em relação aos partos hospitalares imposto pela hegemonia biomédica, seja quebrado e desanuviado.

www.scielo.br/pdf/ean/v17n2/v17n2a14.pdf

5. Brasil, Ministério da Saúde. Atenção ao pré-natal de baixo risco. Série A, Normas e Manuais Técnicos, Caderno de Atenção básica $\mathrm{N}^{\circ} 32$. Secretaria de Atenção à Saúde, Departamento de Atenção Básica. Brasília-DF. 2012; 318 p. Disponível em: http:// bvsms.saude.gov.br/bvs/publicacoes/cadernos_ atencao_basica_32_prenatal.pdf

6. Sanfelice CFO, Abbud FSF, Pregnolatto OS, Silva MG, Shimo AKK. Do parto institucionalizado ao parto domiciliar. Rev Rene [Internet]. 2014 mar; 15(2): 362-370. Disponível em: http://www. redalyc.org/articulo.oa?id=324031263022

7. Collaço VS, Santos EKA, Souza KV, Alves HV, Zampiri MF, Gregório VRP. O significado atribuido pelo casal ao parto domiciliar planejado, assistido pelas enfermeiras obstétricas da Equipe Hanami. Texto \& Contexto Enferm [Internet]. 2017; 26(2): 1-9. Disponível em: http://dx.doi. org/10.1590/0104-07072017006030015

8. Reis RP, Santos RB, Moura RS, Bezerra DG. 
Parto humanizado: compreender para executar. Revista Hórus [Internet]. 2015; 10(1): 133-143. Disponível em: http://periodicos.estacio.br/index. php/revistahorus/article/view/3952

9. Polit DF, Beck CT, Leal SMZ, Toledo MGFS, Lora KVPS. Fundamentos de Pesquisa em Enfermagem: Avaliação de Evidências para a Prática da Enfermagem. 9a ed. São Paulo: Artmed; 2018. $456 \mathrm{p}$.

10. Brasil, Ministério da Saúde. Portaria No20, de 10 de junho de 2014. Secretaria de Ciência, Tecnologia e Insumos Estratégicos. Disponível em: http:// bvsms.saude.gov.br/bvs/saudelegis/sctie/2014/ prt0020_10_06_2014.html

11. Silva SPS, Prates RCG, Campelo BQA. Parto normal ou cesariana? Fatores que influenciam na escolha da gestante. Rev Enferm UFSM [Internet]. 2014; 4(1): 1-9. Disponível em: https://periodicos. ufsm.br/reufsm/article/view/8861/pdf

12. Coxon K, Sandall J, Fulop NJ. How do pregnancy and birth experiences influence planned place of birth in future pregnancies? Findings from a longitudinal, narrative study. Birth [Internet]. 2015; 42(2): 141-148. Disponível em: https:// onlinelibrary.wiley.com/doi/pdf/10.1111/ birt. 12149

13. Nascimento JP, Mattos DV, Matão MEL, Martins CA, Moraes PA. O empoderamento da mulher no parto domiciliar planejado. J Nurs UFPE on line [Internet]. 2016 nov; 10(Suppl. 5): 4182-7. Disponível em: https://periodicos.ufpe.br/revistas/ revistaenfermagem/article/viewFile/11162/12686

14. Klomp T, Jonge A, Hutton EK, Hers S, Lagro-Janssen AL. Perceptions of labour pain management of Dutch primary care midwives: a focus group study. BMC pregnancy childb [Internet]. 2016; 16(1): 1-9. Disponível em: https://bmcpregnancychildbirth.biomedcentral. com/track/pdf/10.1186/s12884-015-0795-6

15. Koettker JG, Brüggemann OM, Knobel R. Resultados maternos dos partos domiciliares planejados assistidos por enfermeiras da equipe hanami no sul do Brasil, 2002-2012. Texto Contexto Enferm [Internet]. 2017; 26(1): 1-11. Disponível em: http://www.scielo.br/pdf/tce/ v26n1/pt_0104-0707-tce-26-01-3110015.pdf

16. Miller T, Smith H. Establishing partnership with traditional birth attendants for improved maternal and newborn health: a review of factors influencing implementation. BMC pregnancy childb [Internet]. 2017; 17(1): 365. Disponível em: https://bmcpregnancychildbirth.biomedcentral. com/track/pdf/10.1186/s12884-017-1534-y

17. Asefa A, Bekele D, Morgan A, Kermode M. Service providers' experiences of disrespectful and abusive behavior towards women during facility based childbirth in Addis Ababa, Ethiopia. Reprod Health [Internet]. 2018; 15(4): 1-9. Disponível em: https://reproductive-health-journal.biomedcentral. com/articles/10.1186/s12978-017-0449-4

18. Ahmad M, Ganji J. The influence of cold pack on labour pain relief and birth outcomes: a randomised controlled trial. J Clin Nurs [Internet]. 2013 nov 11; 23: 2473-2480. Disponível em: https://onlinelibrary.wiley.com/doi/full/10.1111/ jocn. 12413

19. World Health Organization. Recommendations intrapartum care for a positive childbirth experience [Internet]. Switzerland. 200p. Disponível em: http://www.who.int/reproductivehealth/ publications/intrapartum-care-guidelines/en/

20. Koettker JG, Brüggemann OM, Dufloth RM, Monticelli M, Knobel R. Comparaçáo de resultados obstétricos e neonatais entre primíparas e multíparas assistidas no domicílio. Cien enferm [Internet]. 2015; 21(2): 113-125. Disponível em: https:// scielo.conicyt.cl/pdf/cienf/v21n2/art_11.pdf

21. Mselle LT, Kohi TW, Dol J. Barriers and facilitators to humanizing birth care in Tanzania: findings from semi-structured interviews with midwives and obstetricians. Reprod Health [Internet]. 2018; 15(1): 1-10. Disponível em: https:// reproductive-health-journal.biomedcentral.com/ articles/10.1186/s12978-018-0583-7

22. Lessa HF, Tyrrell MAR, Alves VH, Rodrigues DP. A opção pelo parto domiciliar planejado: uma opçáo natural desmedicalizada. Revista de Pesquisa: Cuidado é Fundamental [Internet]. 2018 out/dez; 10(4): 1118-1122. Disponível em: http://www. seer.unirio.br/index.php/cuidadofundamental/ article/view/6341/pdf_1

23. Silva ALS, Nascimento ER, Coelho EAC. Práticas de enfermeiras para promoção da dignificação, participaçáo e autonomia de mulheres no parto normal. Esc Anna Nery [Internet]. 2015; 19(3): 424-431. Disponível em: http://www.scielo.br/ pdf/ean/v19n3/1414-8145-ean-19-03-0424.pdf

24. Studer MA, Smith AE, Lustik MB, Carr MR. Newborn pulse oximetry screening to detect critical congenital heart disease. J Pediatr [Internet]. 2014; 164(3): 505-509. Disponível em: https:/www.sciencedirect.com/science/article/pii/ S0022347613013619

25. Sanfelice CFO, Shimo AKK. Boas práticas em partos domiciliares: perspectiva de mulheres que tiveram experiência de parto em casa. Rev Eletr Enf [Internet]. 2016; Disponível em: https://revistas. ufg.br/fen/article/view/31494/21363

26. Li Y, Townend J, Rowe R, Brocklehurst P, Knight M, Linsell $\mathrm{L}$, et al. Perinatal and maternal outcomes in 
planned home and obstetric unit births in women at 'higher risk' of complications: secondary analysis of the Birthplace national prospective cohort study. BJOG [Internet]. 2015 jan 15; 122(5): 741-753. Disponível em: https://obgyn.onlinelibrary.wiley. com/doi/full/10.1111/1471-0528.13283

27. Good RJ, Canale SK, Goodman RL, Yeager SB. Identification of critical congenital heart disease in Vermont: the role of universal pulse oximetry screening in a rural state. Clin Pediatr [Internet]. 2015 jun 1; 54(6): 570-574. Disponível em: https://journals.sagepub.com/doi/ abs/10.1177/0009922814558247

28. Das A, Nawal D, Singh MK, Karthick M, Pahwa $\mathrm{P}$, Shah $\mathrm{MB}$, et al. Impact of a nursing skillimprovement intervention on newborn-specific delivery practices: An experience from Bihar, India.
Birth [Internet]. 2016 jun 20; 43(4): 328-335. Disponível em: https://onlinelibrary.wiley.com/ doi/full/10.1111/birt.12239

29. Monteiro MCM, Holanda VR, Melo GP. Análise do conceito parto humanizado de acordo com o método evolucionário de Rodgers. Rev Enferm Cent-Oeste Min [Internet]. 2017; Disponível em: http://seer.ufsj.edu.br/index.php/recom/article/ view/1885/1808

30. De Jonge A, Geerts CC, Van Der Goes BY, Mol BW, Buitendijk SE, Nijhuis JG. Perinatal mortality and morbidity up to 28 days after birth among 743 070 low-risk planned home and hospital births: a cohort study based on three merged national perinatal databases. BJOG [Internet]. 2015; 122(5): 720-728. Disponível em: https://doi. org/10.1111/1471-0528.13084 